\title{
Preface to BIT 54:4
}

\section{Axel Ruhe}

Published online: 13 December 2014

(C) Springer Science+Business Media Dordrecht 2014

\section{Collaborators for BIT volume 54}

A new volume is completed, and it is time to say thank you to all, that have made this volume possible, authors, editors, printers and publishers!

But the special thank you is directed to all of you that have helped out as referees. Your work is done voluntarily as a service to the profession, and you are the ones that help us to decide which manuscripts that can be transformed into readable papers. About one manuscript in four makes it, the rest of the authors get some words on the way to get their acts together next time.

Those are the referees reporting from November 1, 2013 to October 31, 2014. Forgive me, if I missed someone deserving to be here:

Ben Adcock

Gregoire Allaire

David Amsallem

Andreas Asheim

Winfried Auzinger

Constantin Bacuta

Zhaojun Bai

Lehel Banjai

John W Barrett

Andrea Barth
Christian Bayer

Roland Becker

Martin Berggren

Jean-Paul Berrut

Timo Betcke

Paolo Bientinesi

Peter Binev

Philipp Birken

Hermann Brunner

Raimund Buerger

A. Ruhe $(\varangle)$

KTH, Stockholm, Sweden

e-mail: ruhe@csc.kth.se 
Carmen Rodrig Coardiel

Mark Carpenter

Fernando Casas

Stephanie Chaillat-Loseille

Long Chen

Feng Chen

Yingda Cheng

Lucas Chesnel

Snorre H. Christiansen

Eric W Chu

Matthias Chung

Xavier Claeys

David Cohen

Eric Darrigrand

Penny Davies

Oleg Davydov

Carl de Boor

Mehdi Dehghan

Laurent Demanet

Richard Di Liu

Josef Dick

Boris Diskin

Froilán M Dopico

Huoyuan Duan

Serge Dubuc

Kenneth Duru

Matthias Ehrhardt

Johannes Elschner

Brittany Erickson

Bengt Fornberg

José María Franco

Walter Gander

Mahadevan Ganesh

Gregor Gassner

Mike Giles

Ron Goldman

Bill Gragg

Thomas Grandine

Volker Grimm

Thomas Hagstrom
Ernst Hairer

Nicholas Hale

Xuli Han

Martin Hanke

Michael Hanke

Antti Hannukainen

Stefan Heinrich

Johan Helsing

Domingo Hernandez-Abreu

Holger Heumann

Hakon Hoel

Johan Hoffman

Weizhang Huang

Daan Huybrechs

Zdzislaw Jackiewicz

Luc Jaulin

Kurt Jetter

Bangti Jin

Shi Jin

Stephen Joe

Fredrik Johansson

Andreas Karageorghis

Lars Karlsson

David I Ketcheson

Misha Kilmer

Othmar Koch

Yoshio Komori

Arne Kovac

Jeremy Kozdon

Gunilla Kreiss

JaEun $\mathrm{Ku}$

Patrick Kuerschner

Elisabet Larsson

Stig Larsson

Raytcho Lazarov

Jeonghun Lee

Richard Lehoucq

Tony Lelievre

Dmitriy Leykekhman

Tiejun Li 
Bernt Lie

Fawang Liu

Tomas Lundquist

Per Lötstedt

Scott Mac Lachlan

Michael Mascagni

Stefano Maset

Marie-Laurence Mazure

Karl Meerbergen

Christian Mehl

Markus Melenk

Michael Minion

Jaun Montijano

Ron Morgan

Paul Muir

Georg Muntingh

Axel Målqvist

Ned Nedialkov

Deanna Needell-Hunter

Michael Neilan

Arnold Neumaier

Serge Nicaise

Harald Niederreiter

Freris Nikolaos

Anna Nissen

Fabio Nobile

Sotirios E Notaris

Yvan Notay

Paolo Novati

Luke Olson

Sheehan Olver

Alexander Ostermann

Brynjulf Owren

Kazufumi Ozawa

Beatrice Paternoster

Clemens Pechstein

Francesca Pelosi

J. M. Pena

Per Pettersson

Martin Plesinger
John Derwent Pryce

Panayiotis Psarrakos

Ronny Ramlau

Joachim Rang

Teresa Reginska

Nils Henrik Risebro

Siegfried M. Rump

Jens Saak

Achim Schaedle

Gabriela Schranz-Kirlinger

Fiorella Sgallari

Meiyue Shao

Philip Sharp

Valeria Simoncini

Alexandra Smirnova

Hendrik Speleers

Nicole Spillane

Rob Stevenson

Martin Stynes

Hai-Wei Sun

Lina von Sydow

Xue-Cheng Tai

Aretha Teckentrup

Dan Tiba

Alex Townsend

Marnix Van Daele

Arthur E P Veldman

Olivier Verdier

James H Verner

Lujun Wang

Tim Warburton

J.A.C. Weideman

Ewa Weinmüller

Holger Wendland

Zhengfu Xu

Jinchao Xu

Hongguo $\mathrm{Xu}$

Qianqian Yang

Chao Yang

Mohsen Zayernouri

Yongtao Zhang 


\section{Introduction to the contents of BIT 54:4}

The papers we collect in this issue have been available online since more than half a year, and we get new contributions ready all the time.

These are the papers:

Winfried Auzinger, Othmar Koch, and Amir Saboor Bagherzadeh study a two point boundary value problem of an ordinary differential equation. They use a locally weighted defect for an error estimate, that can be used for adaptive mesh refinement.

Lehel Banjai and Maryna Kachanovska formulate the three-dimensional wave equation as a time-domain integral equation. It is discretized by a Runge-Kutta based convolution quadrature. The behavior of the kernel of this operator is established, and algorithms to compute the convolution weights are studied.

Alfonso Bueno-Orovio, David Kay, and Kevin Burrage study a Fourier spectral method for fractional-in-space differential equations. These equations are used to model super-diffusion effects in spatially extended structures. The method is applied to the Allen-Cahn equation for movement of phase boundaries, the FitzHugh-Nagumo model for impulse propagation in nerve membranes, and the Gray-Scott model for an autocatalytic chemical reaction.

Costanza Conti, Jean-Louis Merrien, and Lucia Romani describe an algorithm to refine a set of data vectors by repeated application of a subdivision operator to produce a sequence of even denser vector sets. New theoretical results for de Rham type Hermitian subdivisions are derived.

Catterina Dagnino, Sara Remogna, and Paul Sablonnière use spline quasiinterpolating projectors on a bounded interval, for the numerical solution of linear Fredholm integral equations of the second kind. Several algorithms are compared on a set of numerical examples.

Nicholas Hurl, William Layton, Yong Li, and Catalin Trenchea use a Crank Nicolson Leap-Frog method with the Robert Asselin Williams time filter to simulate a geophysical flow. Conditions for stability are established.

Jonas Kiessling and Raul Tempone derive error estimates of a finite difference scheme for option pricing in exponential Lévy models. Expressions are given for the dominating terms in the space and time discretization errors, when the payoff is subject to an exponential growth condition. Small jumps are approximated by a diffusion.

Yoshio Komori and Kevin Burrage derive a stochastic exponential Euler scheme for multi-dimensional, non-commutative stochastic differential equations with semilinear drift terms. Such systems are used in simulation of stiff biochemical reaction systems.

$J a E u n K u$ studies a mixed finite element method for the primary function on unstructured meshes. It is shown that the least squares solutions are higher order perturbations of the solutions obtained by mixed and Galerkin methods. An error estimate for shape regular meshes is derived.

Abdellah Lamnii, Mohamed Lamnii, and Hamid Mraoui derive cubic spline quasiinterpolants on Powell-Sabin partitions. The coefficients of the interpolants depend only on a set of local function values and the interpolants have optimal approximation order.

Yuto Miyatake and Takayasu Matsuo describe a general framework for finding energy dissipative or conservative Galerkin schemes, and their underlying weak forms, 
Preface to BIT 54:4

871

for nonlinear evolution equations. Properties of the discrete partial derivative method are studied to establish its limits, and possible generalizations to semidiscrete disspatine schemes.

Andrew Skelton and Allan R. Willis describe an algorithm for enclosing a given set of time series data by a continuous piecewise linear band of varying height, subject to certain constraints. The band is defined by two piecewise linear curves that lie above and below the data.

That was all! I wish you all a rewarding read,

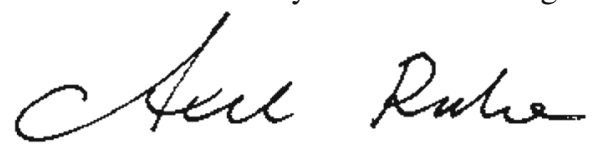

Axel Rube

Springer 